\title{
Two-step Intensity-modulated Radiation Therapy for Oropharyngeal Cancer: Initial Clinical Experience and Validation of Clinical Staging
}

\author{
HITOSHI TATEBE ${ }^{1}$, HIROSHI DOI ${ }^{1}$, KAZUKI ISHIKAWA ${ }^{1}$, HISATO KAWAKAMI ${ }^{2}$, \\ MASAKI YOKOKAWA ${ }^{1}$, KIYOSHI NAKAMATSU ${ }^{1}$, SHUICHI KANAMORI ${ }^{1}$, \\ TORU SHIBATA ${ }^{3}$, MUTSUKAZU KITANO ${ }^{4}$ and YASUMASA NISHIMURA ${ }^{1}$ \\ Departments of ${ }^{1}$ Radiation Oncology, ${ }^{2}$ Medical Oncology, and ${ }^{4}$ Otorhinolaryngology, \\ Kindai University Faculty of Medicine, Osaka, Japan; \\ ${ }^{3}$ Department of Radiation Oncology, Kagawa University Hospital, Kagawa, Japan
}

\begin{abstract}
Aim: To evaluate the clinical results of two-step intensity-modulated radiation therapy (IMRT) for oropharyngeal cancer. Patients and Methods: Eighty patients were treated with two-step IMRT between 2002 and 2014. Whole-neck radiotherapy (44.0-50.0 Gy/22-25 fractions) was delivered by IMRT, followed by boost IMRT to the high-risk clinical target volume (total dose of $70.0 \mathrm{~Gy} / 35$ fractions). Forty-seven patients received concurrent chemotherapy. Immunohistochemistry for human papillomavirus type 16 (HPV/p16) was performed for 64 patients. Results: The 5 -year overall survival and locoregional control rates for stage I, II, III, and IVA-B disease were $80.0 \%, 75.0 \%$, $78.0 \%$, and $64.0 \%$ and $100.0 \%, 75.0 \%, 92.0 \%$, and $82.0 \%$, respectively. Overall survival was significantly higher in HPV/p16-positive patients than in HPV/p16-negative patients $(p=0.01)$. Xerostomia of grade 2 or more was noted in 10 patients. Conclusion: Favourable overall survival and locoregional control rates with excellent salivary preservation were obtained using the two-step IMRT method for oropharyngeal cancer.
\end{abstract}

Radiation therapy (RT) is the main treatment for head and neck cancer (HNC). Patients with locally advanced HNC who are treated with definitive RT have a 5-year survival rate of $40-60 \%$ (1-3). However, long-term late sequelae of

Correspondence to: Hitoshi Tatebe, MD, Department of Radiation Oncology, Kindai University Faculty of Medicine, 377-2 Ohnohigashi, Osaka-Sayama, Osaka 589-8511, Japan. Tel: +81 723660221, Fax: +81 723682388, e-mail: 1615060909@edu.med.kindai.ac.jp

Key Words: Human papillomavirus, intensity-modulated radiation therapy, locoregional control, oropharyngeal cancer, overall survival, radiation therapy.
RT are highly prevalent and have severe adverse effects on the quality of life (4-8). In particular, RT-induced xerostomia is the most prevalent late toxicity for HNC.

Highly conformal RT techniques, such as intensitymodulated RT (IMRT), allow the dose to the surrounding normal tissues to be reduced while maintaining the dose to the target volume. Several studies on IMRT have demonstrated promising locoregional tumour control, as well as, potential preservation of salivary function, swallowing, and quality of life (4-8).

Although IMRT methods vary according to each institution, most institutions use simultaneous integrated boost (SIB) techniques. SIB-IMRT consists of only one treatment plan: 33 fractions of $2.12 \mathrm{~Gy}$ and $1.7 \mathrm{~Gy}$ to the high- and low-risk planning target volumes (PTVs), respectively (9). Although SIB-IMRT is an exciting new technique for improving the therapeutic ratio, there remains a question of whether an initial IMRT plan can be used for the whole course of fractionated RT. As treatment planning and quality assurance of IMRT plans require considerable time to prepare, most investigators use the initial IMRT plan for the whole course of IMRT (4-6). However, significant anatomical changes, including shrinking of the primary tumour or nodal masses, and body weight loss have been reported during fractionated RT for HNC (4-6). In a previous study (6), we also revealed that the volume of the parotid glands had decreased by $26 \%$ during the course of IMRT. These changes in body contour, target volumes, and organs at risk during IMRT can affect the dose distribution to the target volume and organs at risk, which can lead to marginal recurrence or late toxicities (4-6).

In order to avoid changes in the dose distribution during the 7- to 8-week period of IMRT, in this study, a two-step IMRT method was adopted for HNC. For all patients, treatment planning computed tomography was performed before IMRT (CT1) and during the third or fourth week of 
IMRT (CT2) for the boost IMRT treatment planning. Wholeneck RT (44-50 Gy/22-25 fractions) was delivered by IMRT, followed by boost IMRT to the high-risk clinical target volume (CTV) to a total dose of $70 \mathrm{~Gy} / 35$ fractions.

We previously reported the clinical results of two-step IMRT for patients with nasopharyngeal cancer at our institution (4). The aim of this study was to retrospectively evaluate the clinical results of our adaptive RT scheme using a two-step IMRT method for treating patients with oropharyngeal cancer (OPC). In addition, we examined the relationship between human papillomavirus (HPV) status, which is considered a strong prognostic factor, and the clinical results.

\section{Materials and Methods}

Patients and study design. This retrospective study was conducted with Institutional Review Board approval (no. 26-262) and in accordance with the Declaration of Helsinki and Title 45, US Code of Federal Regulations, Part 46, Protection of Human Subjects, effective December 13, 2001. Informed written consent for IMRT as a new method of RT was obtained from all patients.

The clinical results of 80 consecutive patients with OPC who were treated with curative intent by two-step IMRT at our Institution between December 2002 and June 2014 were analyzed. The characteristics of the patients are summarised in Table I. The cohort comprised 59 men and 21 women, with a median age of 66 (range $=35$ 85) years. Pre-treatment evaluations included a complete history and physical examination, routine blood tests, and a laryngoscopy. All patients were examined by gastrointestinal fibroscopy, whole-body computed tomographic (CT) scans with/without head and neck magnetic resonance imaging (MRI). From 2006, 51 patients $(63.8 \%)$ also underwent ${ }^{18} \mathrm{~F}$-fluorodeoxyglucose positron-emission tomography (PET)/CT $(\mathrm{n}=17 ; 21.3 \%)$ or PET/CT simulation scans $(\mathrm{n}=34 ; 42.5 \%)$ $(10,11)$. Staging was performed according to the seventh edition of the tumour-node-metastasis (TNM) classification of malignant tumours (12). All patients were presented at our weekly Tumour Board meeting. Based on the joint recommendations from the multidisciplinary team meeting, patients were selected for RT alone or chemoradiation therapy. Forty-three patients (53.8\%) had undergone ipsilateral or bilateral neck dissection before definitive RT. In addition, volume reduction surgery of the primary tumour was performed in 34 patients $(42.5 \%)$ before RT.

Treatment. Eighty consecutive patients were treated with the twostep IMRT method. The median follow-up period of the surviving patients was 64 (range $=28-134)$ months.

Between 2002 and 2014, 33 patients (41.3\%) with early-stage OPC and major comorbidity or poor performance status were treated with RT alone. Concurrent chemotherapy was administered to 47 patients $(58.8 \%)$. Based on the successive implementation of new treatment strategies during the study period, the concurrent chemotherapy regimen was modified. Between 2003 and 2005, eight patients $(10.0 \%)$ were treated with RT (70.0 Gy) and concomitant weekly docetaxel $\left(10.0-15.0 \mathrm{mg} / \mathrm{m}^{2}\right)$. Between 2005 and 2013, 27 patients $(33.8 \%)$ were treated with RT (70.0 Gy) and three cycles of concomitant cisplatin $\left(80.0 \mathrm{mg} / \mathrm{m}^{2}\right.$ every 3 weeks). Five patients $(6.3 \%)$ with poor renal function were treated with weekly carboplatin (5 areas under the curve). Between 2013 and
2014, six elderly patients $(7.5 \%)$ with poor renal function were treated with weekly cetuximab $\left(250.0 \mathrm{mg} / \mathrm{m}^{2}\right)$. The treatment parameters are summarized in Table II.

Patients were immobilised with a thermoplastic mask covering the head, neck, and shoulders (Type-S thermoplastic-based system; MED-TEC, Orange City, IA, USA). Treatment planning CT scans were obtained with contrast medium at $2.0-\mathrm{mm}$ slice intervals from the head through the aortic arch. For all patients, treatment planning CT was performed before IMRT (CT1) and during the third or fourth week of IMRT (CT2) for boost IMRT. In most instances, a new thermoplastic mask was made for CT2.

The gross tumour volume (GTV) included any visible disease on imaging studies (MRI, CT, or PET/CT) and physical examination $(10,11)$. The primary CTV encompassed a 5.0-10.0 mm margin with appropriate anatomical correction around the primary GTV. The nodal CTV was defined and delineated according to the Danish Head and Neck Cancer Group, European Organisation for Research and Treatment of Cancer, French Group of Radiation Oncology for Head and Neck Cancer, French Head and Neck Cancer Group, National Cancer Institute of Canada, and Radiation Therapy Oncology Group consensus guidelines (13). Cervical and retropharyngeal lymph nodes with the shortest axial diameters of $10 \mathrm{~mm}$ or more and $5 \mathrm{~mm}$ or more, respectively, on CT or MRI were defined as metastatic. Lymph nodes of borderline size with abnormal enhancement were also indications of malignancy. The oropharyngeal region, bilateral level II-IV nodes, and the retropharyngeal nodes were included in the initial CTV. Submandibular lymph nodes (level Ib) were only included in the CTV when involved lymph nodes were suspected in ipsilateral level Ib. Margins of 3.0-4.0 mm for treatment set-up and internal organ motion error were added to the CTV to determine the PTV. For the planning volume for organs at risk, a 3.0-mm margin was added to the spinal cord (14). For the parotid glands, no margin was added in treatment planning.

After whole-neck RT (44-50 Gy/22-25 fractions) was delivered by IMRT, boost IMRT (to a total dose of $70 \mathrm{~Gy} / 35$ fractions) was prescribed to PTV2, which included the GTV and appropriate margins based on CT2. The daily prescribed dose to the PTV was $2 \mathrm{~Gy}$. The prescribed dose was normalised to the dose to $95.0 \%$ of the PTV.

Our goals on dose-volume histogram parameters were maximum PTV $<120 \%$ of the prescribed dose, mean PTV $<105 \%$ (usually $103-$ $104 \%$ ), maximum dose delivered to the spinal cord $<50 \mathrm{~Gy}$, maximum dose delivered to the brain $<70.0 \mathrm{~Gy}$, median dose $<20 \mathrm{~Gy}$, and mean dose $<26$ Gy for at least one parotid gland. The IMRT beam arrangements consisted of seven or nine coplanar beams. Treatment planning for IMRT was performed using inverse planning on commercial treatment planning systems (CadPlan-Helios; Varian Associates Ltd., Palo Alto, CA, USA and Eclipse; Varian Medical Systems International Inc., Baden, Switzerland). IMRT was delivered using dynamic multileaf collimation with one of two linear accelerators (Clinac 600C, Clinac 21EX; Varian Associates Ltd.) equipped with a 40-leaf dynamic multileaf collimator. Beam energy of 4 or $6 \mathrm{MV}$ X-rays was used. The daily treatment time was $15-20$ minutes. To verify the leaf motion of each beam, several quality assurance performance tests were conducted. For patient set-up verification, offline bony anatomy matching was performed using megavoltage imaging before the initial IMRT and boost treatments.

Immunohistochemistry. Immunohistochemistry (IHC) for HPV/p16 expression was performed for $64(80.0 \%)$ out of the 80 patients included in this study. Tumour specimens were obtained during surgery or diagnostic biopsy. One representative paraffin block was 
Table I. Characteristics of patients with squamous cell oropharyngeal carcinoma $(n=80)$ and their tumours.

\begin{tabular}{|c|c|}
\hline Characteristic & Value \\
\hline Median age (range), years & $66(35-85)$ \\
\hline \multicolumn{2}{|l|}{ Gender, n $(\%)$} \\
\hline Male & $59(73.8)$ \\
\hline Female & $21(26.2)$ \\
\hline \multicolumn{2}{|l|}{ PS, n (\%) } \\
\hline \multicolumn{2}{|l|}{$056(70.0)$} \\
\hline \multicolumn{2}{|l|}{$123(28.8)$} \\
\hline \multicolumn{2}{|l|}{$21(1.2)$} \\
\hline \multicolumn{2}{|l|}{ Tumour site, n (\%) } \\
\hline Lateral & $50(62.5)$ \\
\hline Anterior & $16(20.0)$ \\
\hline Superior & $10(12.5)$ \\
\hline Posterior & $4(5.0)$ \\
\hline \multicolumn{2}{|l|}{ TNM stage, n (\%) } \\
\hline \multicolumn{2}{|l|}{ Seventh edition } \\
\hline I & $5(6.2)$ \\
\hline II & $8(10.0)$ \\
\hline III & $14(17.5)$ \\
\hline IVA-B & $53(66.3)$ \\
\hline \multicolumn{2}{|l|}{ Eighth edition } \\
\hline \multicolumn{2}{|l|}{ HPV/p16-positive } \\
\hline I & $23(28.8)$ \\
\hline II & $5(6.2)$ \\
\hline III & $3(3.7)$ \\
\hline \multicolumn{2}{|l|}{ HPV/p16-negative } \\
\hline \multicolumn{2}{|l|}{ I $4(5.0)$} \\
\hline \multicolumn{2}{|l|}{ II $6(7.5)$} \\
\hline \multicolumn{2}{|l|}{ III $9(11.3)$} \\
\hline IVA-B & $30(37.5)$ \\
\hline \multicolumn{2}{|c|}{ HPV/p16 expression status, n (\%) } \\
\hline Positive & $31(38.7)$ \\
\hline Negative & $33(41.3)$ \\
\hline Unknown & $16(20.0)$ \\
\hline \multicolumn{2}{|l|}{ Double cancer, n (\%) } \\
\hline Synchronous & $5(6.2)$ \\
\hline Non-synchronous & $23(28.8)$ \\
\hline
\end{tabular}

HPV, Human papilloma virus; PS, performance status; TNM, tumournode-metastasis classification $(18,19)$.

selected for each tumour. Status for patients without tumour tissue available at Kindai University Hospital (Osaka, Japan) for HPV/p16 staining was defined as unknown.

For specimens obtained between 2002 and 2011, IHC for $\mathrm{HPV} / \mathrm{p} 16$ was performed using a CINtec Histology Kit (MTM Laboratories AG, Heidelberg, Germany) based on the monoclonal antibody E6H4 (15). For specimens obtained between 2011 and 2014, IHC for HPV/p16 was based on the monoclonal anti-human p16INK4a (1H4) mouse IgG. HPV/p16 expression was scored as positive if strong diffuse nuclear and cytoplasmic staining was detected in $>70.0 \%$ of the tumour cells $(16,17)$.

Outcomes. The probability of survival after commencing IMRT was estimated using the Kaplan-Meier method, with significance assessed by the log-rank test. Overall survival (OS) was defined as
Table II. Summary of treatment parameters for patients with squamous cell oropharyngeal carcinoma $(n=80)$.

\begin{tabular}{lc}
\hline Treatment & Value \\
\hline Median RT dose (range), Gya & $70(40-70)$ \\
Concurrent chemotherapy, n (\%) & $47(58.8)$ \\
Docetaxel $\left(15.0 \mathrm{mg} / \mathrm{m}^{2}\right.$ weekly) & $8(10.0)$ \\
Cisplatin $\left(80.0-100.0 \mathrm{mg} / \mathrm{m}^{2}\right.$ every 3 weeks) & $27(33.8)$ \\
Carboplatin $(5 \mathrm{AUC}$ weekly) & $5(6.3)$ \\
5 -FU (700.0 $\left.\mathrm{mg} / \mathrm{m}^{2}\right)$ and cisplatin $\left(70.0 \mathrm{mg} / \mathrm{m}^{2}\right)$ & $1(1.2)$ \\
Cetuximab $\left(250.0 \mathrm{mg} / \mathrm{m}^{2}\right.$ weekly) & $6(7.5)$ \\
\hline
\end{tabular}

5-FU, 5-Fluorouracil; AUC, area under the curve; RT, radiation therapy. apelivered in 2 Gy per fraction.

the time to death from any cause. Events for progression-free survival included locoregional or distant tumour progression and death from any cause. Primary tumour or regional lymph node recurrence were considered events for locoregional control (LRC). After IMRT, LRC and distant progression were evaluated every 34 months for more than 5 years by clinical examination and imaging studies (MRI, CT, and PET/CT). When tumour recurrence or distant metastasis was observed, salvage treatment was mandatory.

Toxicities. Late toxicities were graded 90 days after the commencement of IMRT according to the Common Terminology Criteria for Adverse Events, version 4.0 (18). Xerostomia was recorded as the highest score in the 90-day follow-up period.

\section{Results}

The 3- and 5-year OS and LRC rates of all 80 patients with OPC were $72.0 \%$ and $69.0 \%$, and $89.0 \%$ and $85.0 \%$, respectively. According to the TNM classification (seventh edition) (12), the 5-year OS and locoregional control rates for stage I, II, III, and IVA-B disease were $80.0 \%, 75.0 \%, 78.0 \%$, and $64.0 \%$, and $100.0 \%, 75.0 \%, 92.0 \%$ and $82.0 \%$ respectively (Figure 1a). As of March 2017, 28 patients (35.0\%) had died. Fifteen patients (18.8\%) died of their disease, including three treatment-related deaths (3.8\%). Non-OPC-related mortality, including double cancer, myelodysplastic syndrome, anaplastic anaemia, pneumonia, and cerebral infarction, was observed in 13 patients $(16.2 \%)$. At the time of the last follow-up, the remaining 52 patients $(65.0 \%)$ were alive after a median followup period of 64 (range $=28-134)$ months.

The 5-year LRC rates according to the seventh TNM classification (12) were 100.0\%, $75.0 \%, 92.0 \%$, and $82.0 \%$ for patients with stage I, II, III, and IVA-B disease, respectively (Figure 1b). Although there were 10 locoregional recurrences $(12.5 \%)$, no marginal recurrence at the edge of the PTV was observed. All locoregional recurrences were detected in the PTV region receiving 66.0-70.0 Gy. Isolated distant failures were observed in four patients (5.0\%) and one patient $(1.2 \%)$ developed combined failures. 

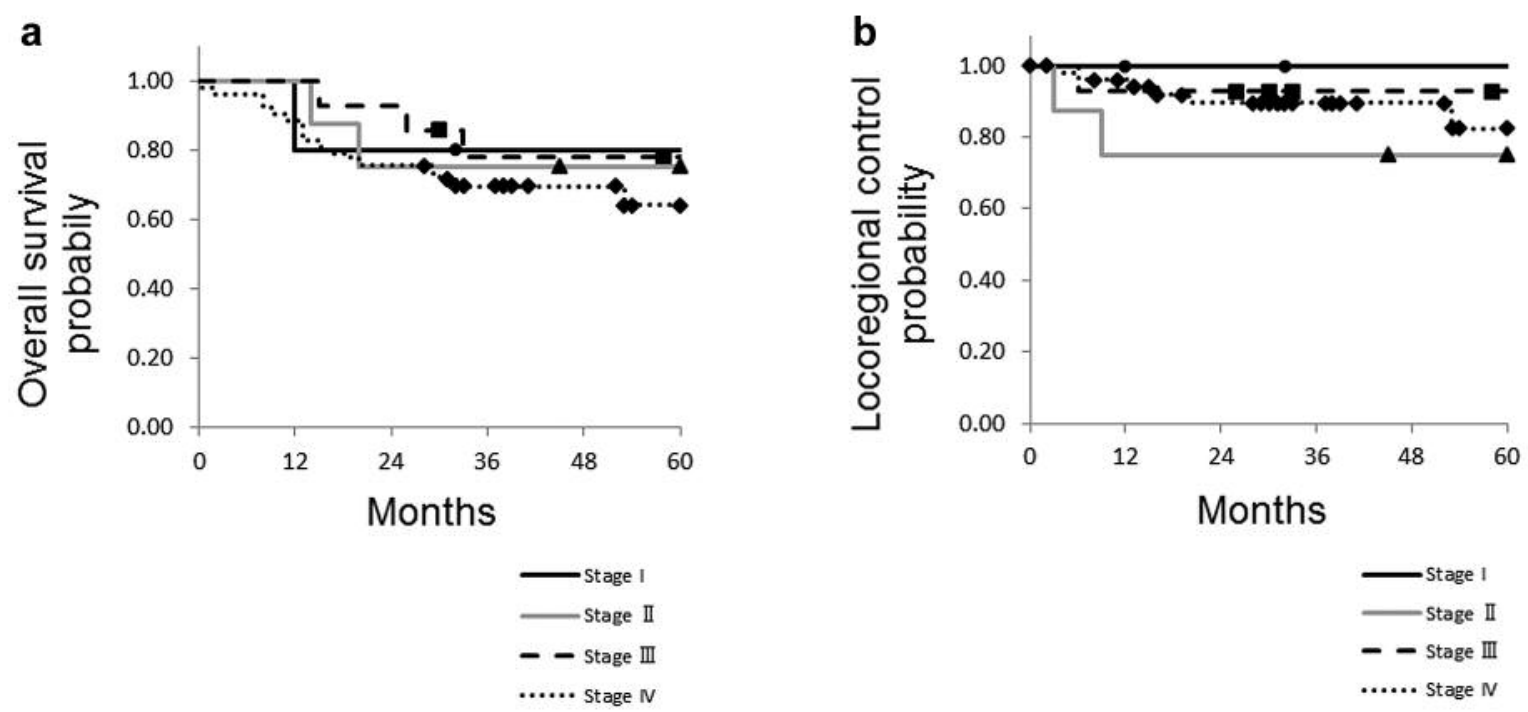

Figure 1. Kaplan-Meier curves of overall survival (a) and locoregional control (b) of patients with oropharyngeal cancer ( $n=80)$ according to stage by the seventh edition of the tumour-node-metastasis classification (18).
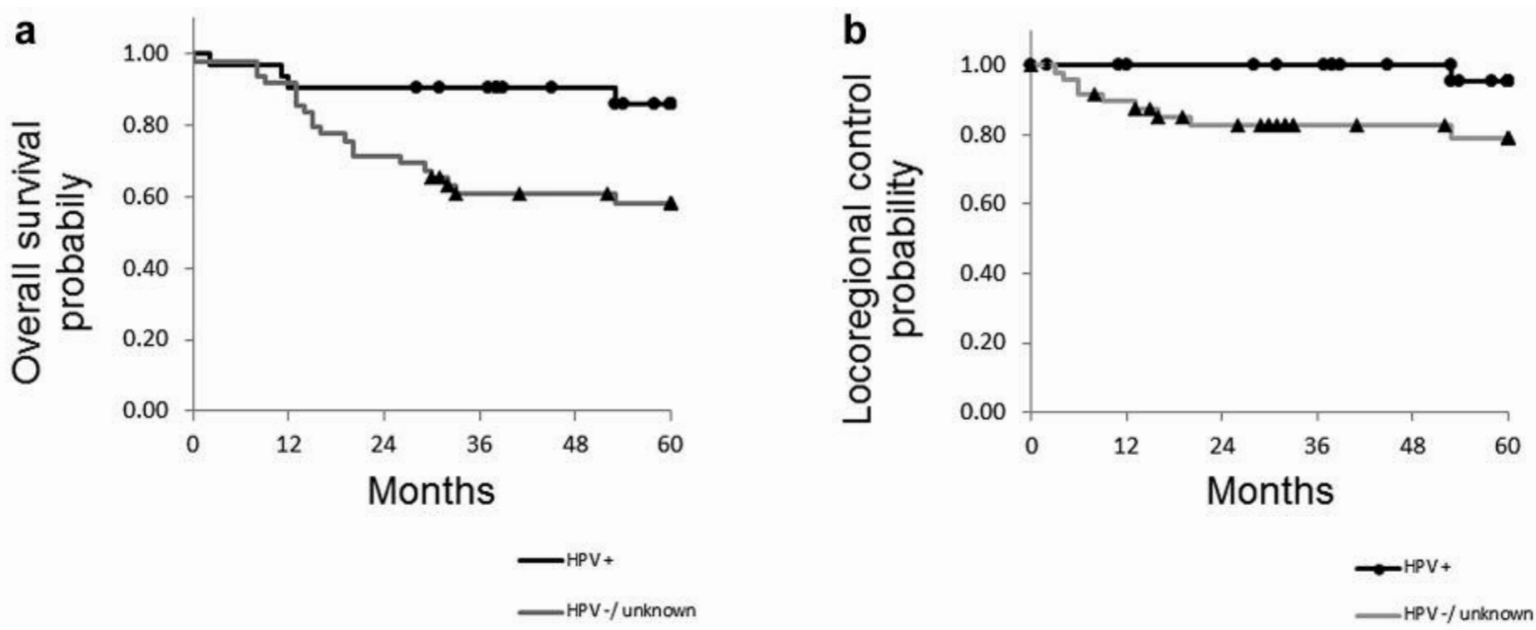

Figure 2. Kaplan-Meier curves of overall survival (a) and locoregional control (b) of patients with oropharyngeal cancer ( $n=80)$ according to human papilloma virus $(\mathrm{HPV}) / \mathrm{p} 16$ expression status.

Thirty-one $(48.4 \%)$ out of the 64 tumour specimens were HPV/p16-positive by IHC analysis. The 5-year OS and LRC rates were significantly higher for patients with $\mathrm{HPV} / \mathrm{p} 16$ positive tumours compared with those with HPV/p16-negative ones or patients in whom IHC analysis was not performed (5year OS: $86.0 \%$ vs. $58.0 \%, p=0.013$ and 5-year LRC: $95.0 \%$ vs. $79.0 \%, p=0.038$ ) (Figure 2). Of the 10 locoregional recurrences, one was $\mathrm{HPV} / \mathrm{p} 16$-positive and seven were $\mathrm{HPV} / \mathrm{p}$ 16-negative. IHC analysis was not performed for the remaining two patients.
Restaging was performed according to the eighth edition of the TNM classification (19). Among the $31 \mathrm{HPV} / \mathrm{p} 16$-positive patients, 23 had stage I, five had stage II, and three had stage III disease. Among the $33 \mathrm{HPV} / \mathrm{p} 16$-negative patients, four had stage I, six had stage II, nine had stage III, and 30 had stage IVA-B disease. For the remaining 16 patients, IHC analysis was not performed. According to staging by the eighth edition of the TNM classification (19), the 5-year OS rates for patients with stage I, II, III, and IVA-B disease were $88.0 \%, 80.0 \%, 66.0 \%$, and $48.0 \%$, respectively (Figure 3a). 

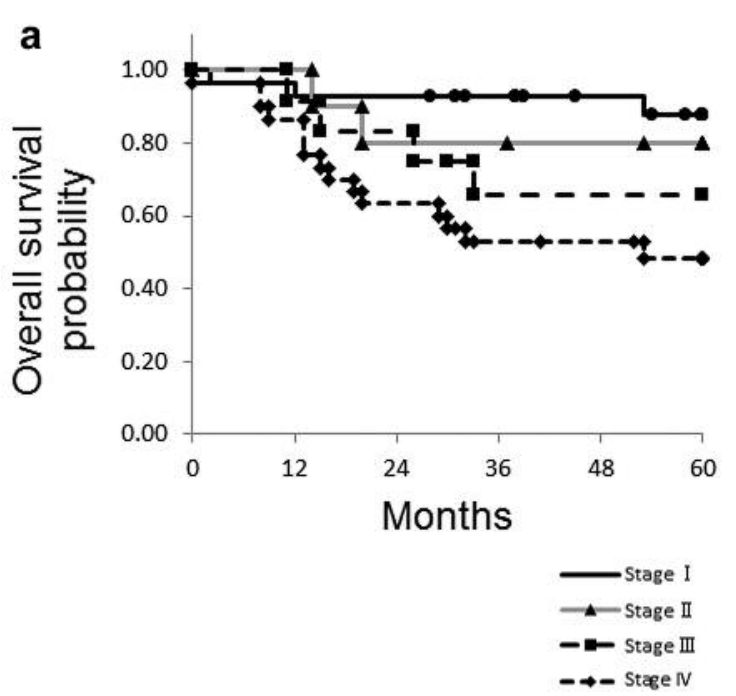
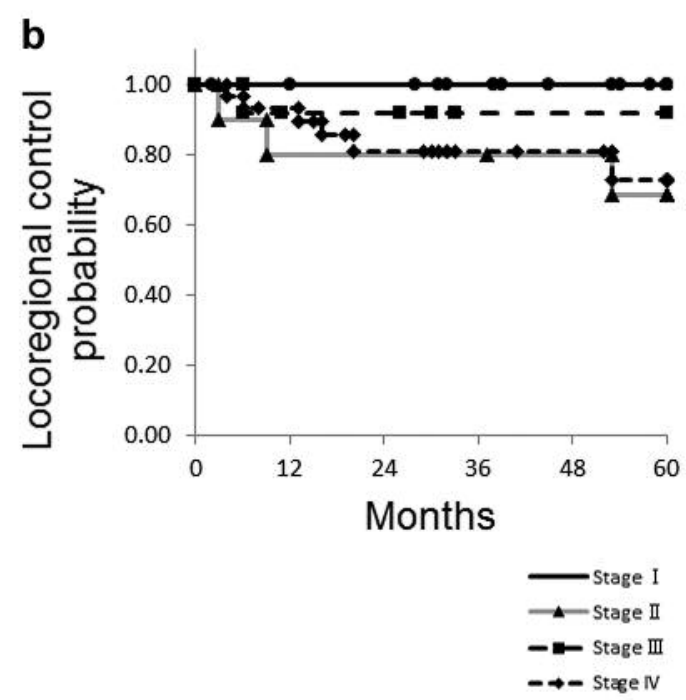

Figure 3. Kaplan-Meier curves of overall survival (a) and locoregional control (b) of patients with oropharyngeal cancer ( $n=80)$ according to stage by the eighth edition of the tumour-node-metastasis classification (19).

The corresponding 5-year LRC rates were 100.0\%, 69.0\%, $92.0 \%$, and $73.0 \%$, respectively (Figure $3 \mathrm{~b}$ ).

Late toxicities associated with IMRT with and without concurrent chemotherapy are summarized in Table III. Hypothyroidism and dysphagia were common late toxicities. Seventeen patients $(21.3 \%)$ complained of dysphagia after treatment, with three $(3.8 \%)$ requiring percutaneous endoscopic gastrostomy. Apparent treatment-related deaths were noted in three patients. One patient died of dysphagia due to severe mucositis. For this patient, RT was terminated at 64.0 Gy (32 fractions) due to grade 4 mucositis. However, persistent mucositis resulted in severe dysphagia, leading to nutritional deficiencies and a deterioration in performance status. One patient who was treated with $66.0 \mathrm{~Gy}$ RT and concomitant weekly docetaxel $\left(15.0 \mathrm{mg} / \mathrm{m}^{2}\right)$ died of carotid artery rupture 74 months after treatment without recurrence. The rupture site involved the primary tumour. The final patient died of infectious pneumonia during IMRT.

Long-standing xerostomia of grade 2 or more was observed in 10 patients $(12.5 \%)$. Excluding patients with early death $(n=2)$, grade 0,1 , and 2 xerostomia at 2 years of treatment were observed in 49, 19, and 10 patients, respectively (Table III).

\section{Discussion}

IMRT has been increasingly adopted as an effective RT technique that provides excellent OS and LRC rates with fewer treatment-related toxicities. Most of the published data on IMRT for the treatment of HNC were collected using the SIB technique. Among the published studies, Huang et al.
Table III. Late toxicities (Common Terminology Criteria for Adverse Events, version 4.0) (18).

\begin{tabular}{lrrrrr}
\hline Toxicity & \multicolumn{5}{c}{ Grade, no. of patients } \\
\cline { 2 - 6 } & G1 & G2 & G3 & G4 & G5 \\
\hline Skin & 10 & 0 & 0 & 0 & 0 \\
Middle ear inflammation & 0 & 2 & 0 & 0 & 0 \\
Dysphagia & 11 & 2 & 3 & 0 & 1 \\
Osteonecrosis of the jaw & 0 & 1 & 1 & 0 & 0 \\
Laryngeal oedema & 0 & 0 & 1 & 0 & 0 \\
Xerostomia at 24 months & 19 & 10 & 0 & - & - \\
Hypothyroidism & 8 & 20 & 0 & 0 & 0 \\
Vascular disorders & 0 & 0 & 0 & 0 & 1 \\
\hline
\end{tabular}

reported 3-year OS and LRC rates of $83 \%$ and $90 \%$, respectively, for patients with stage III and IV OPC using SIB-IMRT (20). Similarly, Daly et al. reported 3-year OS and LRC rates of $83 \%$ and $92 \%$, respectively, for SIB-IMRTtreated patients with OPC (21). These and several other studies (20-27) are summarised in Table IV. These institutions used the SIB technique and produced 3-year OS and LRC rates of approximately $84 \%$ and $90 \%$, respectively. In this study, two-step IMRT was used to treat patients with OPC. The 3-year OS and LRC rates were $72 \%$ and $89 \%$, respectively. Thus, the LRC rate was comparable to that of previous reports. However, the OS rate was slightly lower than that of previous reports. In this study, the HPV/p16positive rate of patients with OPC was approximately $50 \%$. 
Table IV. Summary of reported clinical results of intensity-modulated radiation therapy (IMRT) for oropharyngeal cancer.

\begin{tabular}{|c|c|c|c|c|c|c|c|c|c|}
\hline Study (Ref.) & $\begin{array}{l}\text { Patients } \\
\text { (n) }\end{array}$ & $\begin{array}{l}\text { Median } \\
\text { follow-up } \\
\text { (months) }\end{array}$ & $\begin{array}{c}\text { Stage } \\
\text { (TNM } \\
\text { edition) }\end{array}$ & IMRT & $\begin{array}{l}\text { Dose/ } \\
\text { fx }\end{array}$ & $\begin{array}{l}\text { Adaptive } \\
\text { replan }\end{array}$ & $\begin{array}{c}\text { Xerostomia, } \\
\text { grade }(\%)\end{array}$ & LRC & OS \\
\hline Huang et al. (20) & 71 & 33 & III-IV (6th) & SIB & $70.0 \mathrm{~Gy} / 33$ & - & $2(22 \%)$ & $90 \%(3 y)$ & $83 \%(3 y)$ \\
\hline Daly et al. (21) & 107 & 29 & II-IV (6th) & SIB & $66.0 \mathrm{~Gy} / 30$ & - & - & $92 \%(3 y)$ & $83 \%(3 y)$ \\
\hline Ward et al. (25) & 156 & 22 & I-IV (7th) & SIB & $70.0 \mathrm{~Gy} / 35$ & $42 \%$ & $2(23 \%)$ & $93 \%(2 y)$ & $88 \%(2 y)$ \\
\hline Masoud Rahbari et al. (26) & 61 & 22 & I-IV (7th) & SIB & $70.0 \mathrm{~Gy} / 35$ & - & $1-2(38 \%)$ & $98 \%(2 y)$ & $90 \%(2 y)$ \\
\hline McBride et al. (22) & 132 & 51 & III-IV (6th) & SIB & $70.0 \mathrm{~Gy} / 33$ & - & $1-2(15 \%)$ & $99 \%(5 y)$ & $79 \%(5 y)$ \\
\hline Setton et al. (23) & 404 & 37 & I-IV (5th) & SIB & $70.0 \mathrm{~Gy} / 33$ & - & $2(7 \%)$ & $89 \%(3 y)$ & $85 \%(3 y)$ \\
\hline Garden et al. (24) & 776 & 54 & I-IV (6th) & SIB & $66.0 \mathrm{~Gy} / 33$ & - & - & $90 \%(5 y)$ & $84 \%(5 y)$ \\
\hline Bird et al. (27) & 177 & 26 & I-IV (7th) & SIB & $65.0 \mathrm{~Gy} / 30$ & - & $2(38 \%)$ & $88 \%(3 y)$ & $77 \%(3 y)$ \\
\hline Present series & 80 & 64 & I-IV (7th) & Two-step & $70.0 \mathrm{~Gy} / 35$ & $100 \%$ & $2(12 \%)$ & $85 \%(5 y)$ & $69 \%(5 y)$ \\
\hline
\end{tabular}

Ref., Reference; fx, fractions; LRC, locoregional control; OS, overall survival; SIB, simultaneous integrated boost; y, years.

A recent study in America reported HPV/p16-positive rates as high as $70-80 \%$ (28). In Sweden, the HPV/p16-positive rate of OPC steadily increased over time to $93 \%$ in 2007 (29). This difference in the HPV/p16-positive rate of OPC may have contributed to the slightly lower OS rate in the present study. Notably, the 5-year OS and LRC rates for $\mathrm{HPV} / \mathrm{p} 16$-positive patients were $86 \%$ and $95 \%$, respectively.

For the SIB technique, most investigators use the initial IMRT plan for the whole course of IMRT. Anatomical changes, including shrinking of the primary tumour or nodal masses, and body weight loss during IMRT with/without concurrent chemotherapy have been reported (4-6). The twostep IMRT method may be adaptable to anatomical changes since all patients require re-planning during the third or fourth week of IMRT. However, the two-step IMRT method may lead to marginal recurrences due to insufficient target delineation. Chen et al. reported on the potential negative impact of contouring errors on the prognosis of HPV/p16positive patients with OPC (30). Indeed, at our Institution, we have experienced several cases of early marginal recurrence in patients with nasopharyngeal cancer who were treated by two-step IMRT (4). In the present series of patients with OPC, no marginal recurrences were observed at the edge of the PTV. At our Institution, integrated PET/CT simulation scans have been performed from $2006(9,10)$. These PET/CT simulation scans are especially effective for visualising the GTV, and there is the potential for PET/CT simulation to reduce marginal recurrence (31). In addition, HPV/p16-positive OPC may be characterised by more rapid progression than HPV/p16-negative OPC (32). Overall, our two-step IMRT method may be useful for treating rapidly progressive HPV/p16-positive OPC.

In this series, three patients (3.8\%) died of myelodysplasia or anaplastic anaemia. Recently, RT and chemotherapy were reported to be more likely to increase the risk of therapy- related myeloid neoplasms, including myelodysplasia $(33,34)$. These three cases may be therapy-related myeloid neoplasms influenced by RT with/without concurrent chemotherapy.

Only $10(12.5 \%)$ out of the 80 patients in the current series developed grade 2 xerostomia. None of the patients developed grade 3 or more xerostomia. In our previous studies, we reported on the position and volume changes of the parotid glands during IMRT with and without concurrent chemotherapy $(5,6)$. The two-step IMRT method may be adjusted to anatomical changes, thereby preventing increases in the high-dose regions of the parotid glands (6). The incidence of grade 1-2 xerostomia using the SIB-IMRT method is reported to range from $7-38 \%$, with a median of $22-23 \%$ (20-27) (Table IV). In this series, the rate of grade 2 xerostomia at 24 months was $12.5 \%$. Thus, the effectiveness of the twostep IMRT method for preventing xerostomia was confirmed.

This study has several limitations, including its retrospective single-centre design and limited sample size. However, the treatment protocol of RT was kept constant in this study. In addition, we performed re-staging using the eighth edition of the TNM classification (19) of all analysed patients in order to update the outcomes according to the latest edition of the staging system. In the results, the updated staging system was validated to predict the clinical outcomes after two-step IMRT. The eighth edition of the TNM classification was recently reported to correlate prognosis of OPC better than the seventh edition (35). To our knowledge, this is the first report to describe the clinical results of IMRT for OPC with re-staging using the eighth edition of the TNM classification (19). Another limitation of this study is the lack of physical validation, such as study planning and dosevolume histogram analysis. However, we have already reported the superiority of the two-step IMRT method as an adaptive RT scheme in patients with nasopharyngeal cancer and performed a detailed analysis of the dose parameters for 
the two-step IMRT method in patients with $\operatorname{HNC}(4,6)$. Thus, we decided to use the two-step IMRT method in clinical practice. A prospective comparison study may be ethically challenging at our Institution. Therefore, we believe that our data are sufficiently reliable and could form the basis for future prospective clinical trials in order to directly compare these two different methods of IMRT (SIB and two-step) in combination with updated chemotherapy techniques, including targeted agents and immunotherapeutic approaches.

In conclusion, favourable OS and LRC rates with excellent salivary preservation were obtained using the twostep IMRT method for OPC. No marginal recurrence was detected at the edge of the PTV. Two-step IMRT may be the ideal method of adaptive RT for OPC. In addition, the eighth edition of the TNM classification predicted the clinical outcomes of patients treated with two-step IMRT better than the seventh.

\section{Acknowledgements}

This study was supported, in part, by the National Cancer Center Research and Development Fund (Grant No. 29-A-3) and a Grantin-Aid for Scientific Research from the Japanese Ministry of Education, Culture, Sports, Science, and Technology (Grant No. 16K10406).

\section{References}

1 Lohaus F, Linge A, Tinhofer I, Budach V, Gkika E, Stuschke M, Balermpas P, Rödel C, Avlar M, Grosu AL, Abdollahi A, Debus J, Bayer C, Belka C, Pigorsch S, Combs SE, Mönnich D, Zips D, von Neubeck C, Baretton GB, Löck S, Thames HD, Krause M, Baumann M; DKTK-ROG: HPV16 DNA status is a strong prognosticator of loco-regional control after postoperative radiochemotherapy of locally advanced oropharyngeal carcinoma: results from a multicentre explorative study of the German Cancer Consortium Radiation Oncology Group (DKTK-ROG). Radiother Oncol 113: 317-323, 2014.

2 Leemans CR, Braakhuis BJ and Brakenhoff RH: The molecular biology of head and neck cancer. Nat Rev Cancer 11: 9-22, 2011.

3 Ferlay J, Shin HR, Bray F, Forman D, Mathers C and Parkin DM: Estimates of worldwide burden of cancer in 2008. GLOBOCAN 2008. Int J Cancer 127: 2893-2917, 2010.

4 Nishimura Y, Shibata T, Nakamatsu K, Kanamori S, Koike R, Okubo M, Nishikawa T, Tachibana I, Tamura M and Okumura M: A two-step intensity-modulated radiation therapy method for nasopharyngeal cancer: The Kinki University experience. Jpn J Clin Oncol 40: 130-138, 2010.

5 Nishimura Y, Nakamatsu K, Shibata T, Kanamori S, Koike R, Okumura M and Suzuki M: Importance of the initial volume of parotid glands in xerostomia for patients with head and neck cancers treated with IMRT. Jpn J Clin Oncol 35: 375-379, 2005.

6 Nishi T, Nishimura Y, Shibata T, Tamura M, Nishigaito $\mathrm{N}$ and Okumura M: Volume and dosimetric changes and initial clinical experience of a two-step adaptive intensity modulated radiation therapy (IMRT) scheme for head and neck cancer. Radiother Oncol 106: 85-89, 2013.
7 Nutting CM, Morden JP, Harrington KJ, Urbano TG, Bhide SA, Clark C, Miles EA, Miah AB, Newbold K, Tanay M, Adab F, Jefferies SJ, Scrase C, Yap BK, A'Hern RP, Sydenham MA, Emson M, Hall E; PARSPORT trial management group: Parotidsparing intensity modulated versus conventional radiotherapy in head and neck cancer (PARSPORT): a phase 3 multicentre randomised controlled trial. Lancet Oncol 12: 127-136, 2011.

8 van Rij CM, Oughlane-Heemsbergen WD and Ackerstaff AH, Lamers EA, Balm AJ and Rasch CR: Parotid gland-sparing IMRT for head and neck cancer improves xerostomia related quality of life. Radiat Oncol 3: 41, 2008.

9 Songthong AP, Kannarunimit D, Chakkabat C and Lertbutsayanukul C: A randomized phase II/III study of adverse events between sequential (SEQ) versus simultaneous integrated boost (SIB) intensity-modulated radiation therapy (IMRT) in nasopharyngeal carcinoma; preliminary result on acute adverse events. Radiat Oncol 10: 166, 2015.

10 Okubo M, Nishimura Y, Nakamatsu K, Okumura M, Shibata T, Kanamori S, Hanaoka K and Hosono M: Static and moving phantom studies for radiation treatment planning in a positronemission tomography and computed tomography (PET/CT) system. Ann Nucl Med 22: 579-586, 2008.

11 Okubo M, Nishimura Y, Nakamatsu K, Okumura M, Shibata T, Kanamori S, Hanaoka K and Hosono M: Radiation treatment planning using positron-emission and computed tomography for lung and pharyngeal cancers: a multiple-threshold method for [(18)F]fluoro-2-deoxyglucose activity. Int J Radiat Oncol Biol Phys 77: 350-356, 2010.

12 Sobin LH, Gospodarowicz MK and Wittekind C (eds.): TNM Classification of Malignant Tumours, seventh edition. International Union Against Cancer. New York, Wiley-Blackwell, 2009.

13 Grégoire V, Levendag P, Ang KK, Bernier J, Braaksma M, Budach V, Chao C, Coche E, Cooper JS, Cosnard G, Eisbruch A, El-Sayed S and Emami B, Grau C, Hamoir M, Lee N, Maingon P, Muller K and Reychler H: CT-based delineation of lymph node levels and related CTVs in the node-negative neck: DAHANCA, EORTC, GORTEC, NCIC, RTOG consensus guidelines. Radiother Oncol 69: 227-236, 2003.

14 Suzuki M, Nishimura Y, Nakamatsu K, Okumura M, Hashiba H, Koike R, Kanamori S and Shibata T: Analysis of interfractional set-up errors and intrafractional organ motions during IMRT for head and neck tumors to define an appropriate planning target volume (PTV) and planning organs at risk volume (PRV) margins. Radiother Oncol 78: 283-290, 2006.

15 Kawakami H, Okamoto I, Terao K, Sakai K, Suzuki M, Ueda S, Tanaka K, Kuwata K, Morita Y, Ono K, Nishio K, Nishimura Y, Doi $\mathrm{K}$ and Nakagawa K: Human papillomavirus DNA and p16 expression in Japanese patients with oropharyngeal squamous cell carcinoma. Cancer Med 2: 933-941, 2013.

16 Begum S, Gillison ML, Ansari-Lari MA and Shah K and Westra WH: Detection of human papillomavirus in cervical lymph nodes: a highly effective strategy for localizing site of tumor origin. Clin Cancer Res 9: 6469-6475, 2003.

17 Lassen P, Primdahl H, Johansen J, Kristensen CA, Andersen E, Andersen LJ, Evensen JF, Eriksen JG, Overgaard J; Danish Head and Neck Cancer Group (DAHANCA): Impact of HPVassociated p16-expression on radiotherapy outcome in advanced oropharynx and non-oropharynx cancer. Radiother Oncol 113: 310-316, 2014. 
18 National Cancer Institute (2009) Common Terminology Criteria for Adverse Events (CTCAE). Version 4.0. US Department of Health and Human Services. National Institute of Health. Available https://ctep.cancer.gov/protocolDevelopment/electronic_applicati ons/ctc.htm\#ctc_40 [Accessed 17th October 2017].

19 Brierley JD, Gospodarowicz MK and Wittekind C (eds.): TNM Classification of Malignant Tumours, eighth edition. International Union Against Cancer. New York, Wiley-Blackwell, 2016.

20 Huang K, Xia P, Chuang C, Weinberg V, Glastonbury CM, Eisele DW, Lee NY, Yom SS, Phillips TL and Quivey JM: Intensity-modulated chemoradiation for treatment of stage III and IV oropharyngeal carcinoma: The University of CaliforniaSan Francisco experience. Cancer 113: 497-507, 2008.

21 Daly ME, Le QT, Maxim PG, Loo BW Jr., Kaplan MJ, Fischbein NJ, Pinto $\mathrm{H}$ and Chang DT: Intensity-modulated radiotherapy in the treatment of oropharyngeal cancer: clinical outcomes and patterns of failure. Int J Radiat Oncol Biol Phys 76: 1339-1346, 2010.

22 McBride SM, Parambi RJ, Jang JW, Goldsmith T, Busse PM and Chan AW: Intensity-modulated versus conventional radiation therapy for oropharyngeal carcinoma: long-term dysphagia and tumor control outcomes. Head Neck 36: 492-498, 2014.

23 Setton J, Caria N, Romanyshyn J, Koutcher L, Wolden SL, Zelefsky MJ, Rowan N, Sherman EJ, Fury MG, Pfister DG, Wong RJ, Shah JP, Kraus DH, Shi W, Zhang Z, Schupak KD, Gelblum DY, Rao SD and Lee NY: Intensity-modulated radiotherapy in the treatment of oropharyngeal cancer: an update of the Memorial Sloan-Kettering Cancer Center experience. Int J Radiat Oncol Biol Phys 82: 291-298, 2012.

24 Garden AS, Dong L, Morrison WH, Stugis EM, Glisson BS, Frank SJ, Beadle BM, Gunn GB, Schwartz DL, Kies MS, Weber RS, Ang KK and Rosenthal DI: Patterns of disease recurrence following treatment of oropharyngeal cancer with intensitymodulated radiation therapy. Int J Radiat Oncol Biol Phys 85: 941-947, 2013.

25 Ward MC, Ross RB, Koyfman SA, Lorenz R, Lamarre ED, Scharpf J, Burkey BB, Joshi NP, Woody NM, Prendes B, Houston N, Reddy CA, Greskovich JF and Adelstein DJ: Modern image-guided intensity-modulated radiotherapy for oropharynx cancer and severe late toxic effects: Implications for clinical trial design. JAMA Otolaryngol Head Neck Surg 142: 1164-1170, 2016

26 Masoud Rahbari R, Winkley L, Hill J, Tahir AR, McKay M, Last A, Shakespeare TP and Dwyer P: Definitive intensity-modulated radiotherapy concurrent with systemic therapy for oropharyngeal squamous cell carcinoma: Outcomes from an integrated regional Australian cancer centre. J Med Imaging Radiat Oncol 60: 414419,2016
27 Bird T, De Felice F, Michaelidou A, Thavaraj S, Jeannon JP, Lyons A, Oakley R, Simo R, Lei M, Guerrero Urbano T: Outcomes of intensity-modulated radiotherapy as primary treatment for oropharyngeal squamous cell carcinoma - a European single-institution analysis. Clin Otolaryngol 42: 115122, 2017.

28 Gondim DD, Haynes W, Wang X, Chernock RD, El-Mofty SK and Lewis JS Jr.: Histologic typing in oropharyngeal squamous cell carcinoma: A 4-year prospective practice study with p16 and high-risk HPV mRNA testing correlation. Am J Surg Pathol 40: 1117-1124, 2016.

29 Blomberg M, Nielsen A, Munk C and Kjaer SK: Trends in head and neck cancer incidence in Denmark, 1978-2007: focus on human papillomavirus associated sites. Int J Cancer 129: 733$741,2011$.

30 Chen AM, Chin R, Beron P, Yoshizaki T, Mikaeilian AG and Cai $\mathrm{M}$ : Inadequate target volume delineation and local-regional recurrence after intensity-modulated radiotherapy for human papillomavirus-positive oropharynx cancer. Radiother Oncol 123: 412-418, 2017

31 Matsuura T, Nishimura Y, Nakamatsu K, Kanamori S, Ishikawa K, Tachibana I, Hosono M and Shibata T: Clinical outcomes of IMRT planned with or without PET/CT simulation for patients with pharyngeal cancers. Int J Clin Oncol 22: 52-58, 2017.

32 Chen AM, Li J, Beckett LA, Zhara T, Farwell G, Lau DH, Gandour-Edwards R and Vaughan AT and Purdy JA: Differential response rates to irradiation among patients with human papillomavirus positive and negative oropharyngeal cancer. Laryngoscope 123: 152-157, 2013.

33 Nardi V, Winkfield KM, Ok CY, Niemierko A, Kluk MJ, Attar EC, Garcia-Manero G and Wang SA and Hasserjian RP: Acute myeloid leukemia and myelodysplastic syndromes after radiation therapy are similar to de novo disease and differ from other therapy-related myeloid neoplasms. J Clin Oncol 30: 2340-2347, 2012.

34 Sun LM, Lin CL, Lin MC, Liang JA and Kao $\mathrm{CH}$ : Radiotherapy- and chemotherapy-induced myelodysplasia syndrome: a nationwide population-based nested case-control study. Medicine 94: e737, 2015.

35 Würdemann N, Wagner S, Sharma SJ, Prigge ES, Reuschenbach M, Gattenlöhner S, Klussman JP and Wittekindt C: Prognostic Impact of AJCC/UICC 8th Edition New Staging Rules in Oropharyngeal Squamous Cell Carcinoma. Front Oncol 7: 129, 2017.

Received November 2, 2017

Revised November 27, 2017 Accepted November 28, 2017 